\title{
The effects of exercise and neuromuscular electrical stimulation in subjects with knee osteoarthritis: a 3-month follow-up study
}

This article was published in the following Dove Press journal:

Clinical Interventions in Aging

17 July 2014

Number of times this article has been viewed

\author{
Yocheved Laufer' \\ Haim Shtraker ${ }^{2}$ \\ Michal Elboim Gabyzon' \\ 'Physical Therapy Department, \\ Faculty of Social Welfare and Health \\ Sciences, University of Haifa, Israel; \\ ${ }^{2}$ Department Orthopaedics A and \\ Pediatric Orthopaedics, Western \\ Galilee Medical Center, Nahariya, \\ Israel
}

Background: Strengthening exercises of the quadriceps femoris muscle (QFM) are beneficial for patients with knee osteoarthritis (OA). Studies reporting short-term effects of neuromuscular electrical stimulation (NMES) of the QFM in this population support the use of this modality as an adjunct treatment. The objectives of this follow-up study are to compare the effects of an exercise program with and without NMES of the QFM on pain, functional performance, and muscle strength immediately posttreatment and 12 weeks after completion of the intervention.

Methods: Sixty-three participants with knee OA were randomly assigned into two groups receiving 12 biweekly treatments: An exercise-only program or an exercise program combined with NMES.

Results: A significantly greater reduction in knee pain was observed immediately after treatment in the NMES group, which was maintained 12 weeks postintervention in both groups. Although at this stage NMES had no additive effect, both groups demonstrated an immediate increase in muscle strength and in functional abilities, with no differences between groups. Although the improvements in gait velocity and in self-report functional ability were maintained at the follow-up session, the noted improvements in muscle strength, time to up and go, and stair negotiation were not maintained.

Conclusion: Supplementing an exercise program with NMES to the QFM increased pain modulation immediately after treatment in patients with knee OA. Maintenance of the positive posttreatment effects during a 12-week period was observed only for pain, self-reported functional ability, and walk velocity, with no difference between groups.

Clinical rehabilitation effect: The effects of a comprehensive group exercise program with or without NMES are partially maintained 12 weeks after completion of the intervention. The addition of NMES is recommended primarily for its immediate effect on pain. Further studies are necessary to determine the effects of repeated bouts of exercise with and without NMES in this population.

Keywords: osteoarthritis, neuromuscular electrical stimulation, exercise, follow-up

\section{Introduction}

Knee osteoarthritis (OA) is one of the most common chronic joint diseases affecting older adults worldwide, leading to progressive disability. ${ }^{1}$ The number of adults with knee OA and associated activity limitation is expected to rise substantially with the increase in life expectancy. This will have a significant effect on individuals, the health care system, and society in general; thus, the need for cost-effective treatments is clear. ${ }^{1}$
Correspondence: Yocheved Laufer Physical Therapy Department, University of Haifa, 31905 Haifa, Israel Tel +97248249951

Fax +97248288090

Email yocheved@research.haifa.ac.il 
There is unanimous agreement that exercise is beneficial for patients with $\mathrm{OA}$, and evidence-based clinical practice guidelines stress the key role of therapeutic exercise as a treatment modality for knee OA. ${ }^{2,3}$ Studies indicate that effective exercise programs should include strengthening of the knee musculature, and in particular the quadriceps femoris muscle (QFM), ${ }^{2}$ which has been shown to be weak in subjects with knee OA. ${ }^{4}$ QFM weakness increases joint stress by diminishing attenuation of loads across the joint ${ }^{5}$ and has been shown to be related to both the etiology and progression of knee OA. ${ }^{5,6}$ A meta-analysis that reviewed 22 studies on the effectiveness of QFM strengthening exercises in subjects with knee OA concluded that such exercises improve QFM strength, reduce knee pain, and improve function and quality of life. ${ }^{7}$ However, there are still insufficient data regarding the long-term efficacy of exercise programs for knee OA and the optimal treatment protocols (eg, type of exercise and dosage)., ${ }^{2,7}$

Neuromuscular electrical stimulation (NMES) entails the use of a low-amplitude electrical pulse to induce involuntary muscle contractions. ${ }^{8}$ NMES has been shown to improve QFM strength in healthy individuals and in subjects with various pathological knee conditions, such as after reconstruction of the anterior cruciate ligament ${ }^{9}$ and after total knee arthroplasty. ${ }^{10}$ The advantage of NMES therapy may lie in activation of type 2 muscle fibers at relatively low-contraction intensities. ${ }^{8}$ Furthermore, it is suggested that NMES may overcome central activation failure by bypassing voluntary inhibitions. ${ }^{11}$ The effect of NMES on the QFM of patients with OA has recently received some attention, indicating its potential as a treatment modality for this population. ${ }^{12-17}$ These findings are supported by our previously published study, which compared the immediate effects of a group exercise program delivered with or without NMES to the QFM. ${ }^{18}$

However, it remains unclear whether the beneficial effects of exercise, and particularly the additive effects of NMES, are sustained 3 months after completion of a comprehensive exercise program. The primary objectives of the present study are to compare the short- and long-term effects of a comprehensive group exercise program with or without NMES to the QFM on functional capabilities, QFM performance, and pain of individuals with knee OA.

\section{Materials and methods Subjects}

Individuals referred during a 15-month period to an outpatient physical therapy clinic with radiographic evidence of knee osteoarthritis at a grade II or higher, according to the Kellgren and Lawrence classification (with definite osteophytes and possible narrowing of joint space in at least one compartment $)^{19}$ were screened by an orthopedist specializing in knee surgery. Included were subjects who met the following criteria: age 50 years and older, knee pain for at least 3 months, ability to ambulate independently for at least $10 \mathrm{~m}$, no pacemaker, absence of a medical condition that could affect functional performance, and no knee joint injection in the previous 6 months.

The study was approved by the Helsinki Committee of the Emek Medical Center, and all participants signed an informed consent form before participation. Using concealed envelopes, participants were randomly assigned to one of two treatment groups. All the participants were evaluated before commencement of treatment, immediately after 12 treatment sessions, and during a follow-up assessment session 12 weeks after treatment completion.

\section{Assessment and treatment}

A detailed description of the outcome measures and the two treatment protocols employed in our study has been previously published, presenting the immediate posttreatment effects. ${ }^{18}$ Although the person conducting the exercise program was blind to treatment allocation, blindness of the individual conducting the assessments was not maintained. Following is a brief summary of the research methodology.

The following outcome measures were determined in each evaluation session in a fixed order. Knee pain during the previous week was assessed subjectively by the patients with a visual analog scale (VAS), ${ }^{20}$ and subjective functional performance was evaluated using the Hebrew translated version of the Western Ontario and McMaster Universities Osteoarthritis Index (WOMAC). ${ }^{21}$ Three functional performance tests were implemented at a self-selected, comfortable pace: $10 \mathrm{~m}$ walk test (10 MW), the timed up-and-go test (TUG), and the stair test. Each test was repeated three times, with the mean time, as measured with a digital stopwatch, used for subsequent analyses. Strength of the QFM voluntary isometric contraction (MVIC) and of the QFM voluntary activation was assessed using standardized procedures. Both tests used a validated computerized myometer system (QMA Systems; Gainesville, GA, USA). ${ }^{22}$ Subjects were seated in a specially designed chair that prevented trunk, pelvis, and thigh movement during the QFM contraction. A high-voltage constant current simulator (Digitimer DS7AH Ltd; Welwyn Garden City, United Kingdom) was used to determine central activation. To perform this test, a single rectangular pulse 
of 10 milliseconds duration was delivered at 1,000 mA current intensity both to the resting muscle and during MVIC. Voluntary activation was calculated by dividing the torque increment caused by the stimulus delivered during the MVIC and the torque increment caused by the same stimulus applied to the resting muscle. ${ }^{23}$

All subjects participated in a group exercise program delivered biweekly. In addition, subjects in the electrical stimulation group (NMES) received a series of electrical stimulations to the QFM of the involved leg. Ten contractions were delivered at each session, at maximal tolerated intensity, for a total of 12 sessions.

\section{Statistical analysis}

Descriptive statistics (means, 95\% confidence interval) were calculated for all outcome measures per group and assessment period. Pretreatment comparisons between groups were conducted to determine equivalence, using analysis of variance (ANOVA). Analyses of treatment effects were performed as repeated measures ANOVA, with group, time, and group $\times$ time interaction as factors. Tukey's post hoc tests were performed when the ANOVA indicated a significant effect. The Likelihood Ratio chi-square was used to determine group differences in patient compliance and long-term attendance. Significance was set at $P \leq 0.05$. Statistical analyses were performed using JMP (SAS Institute Inc., Cary, NC, USA).

\section{Results}

A total of 134 patients with a diagnosis of knee OA were screened. Fifty-two women and eleven men met the inclusion/ exclusion criteria. A summary of the demographic characteristics of all subjects is presented in Table 1. There were no differences between groups in terms of demographic characteristics or in terms of baseline outcomes measures. Thirteen subjects did not complete the study; however, there were no differences between noncompliant subjects from either intervention group in terms of baseline characteristics (Figure 1).

Table I Subject characteristics by group (mean \pm standard deviation)

\begin{tabular}{lll}
\hline Characteristic & Exercise $(\mathbf{n}=\mathbf{2 5})$ & NMES $(\mathbf{n}=\mathbf{2 5})$ \\
\hline Female:male, $\mathrm{n}$ & $21: 4$ & $21: 4$ \\
Age, years & $69.4 \pm 7.7$ & $68.3 \pm 7.7$ \\
Height, cm & $159.6 \pm 6.7$ & $162.2 \pm 7.1$ \\
Weight, kg & $77.9 \pm 15.4$ & $81.2 \pm 16.6$ \\
BMl, kg/m ${ }^{2}$ & $30.5 \pm 5.3$ & $31.4 \pm 6.7$ \\
OA duration, years & $4.5 \pm 6.0$ & $4.9 \pm 6.1$ \\
\hline
\end{tabular}

Abbreviations: NMES, neuromuscular electrical stimulation group; BMI, body mass index; OA, osteoarthritis.
A high percentage of patients attended the follow-up appointment 12 weeks after completion of the treatment ( $84 \%$ in the NMES group and $92 \%$ in the exercise group), with no significant difference between groups. No adverse reactions to either treatment were reported.

Descriptive results (means and 95\% confidence intervals), as well as the results of the ANOVA of all outcomes measures are presented in Tables 2 and 3, and in Figures 2-4. A significant group effect was demonstrated only for pain $(P=0.02)$, indicating a greater decrement in pain in the group receiving NMES in addition to an exercise program compared with the exercise-alone group. A significant time effect, indicating overall improvements, was demonstrated for all variables $(P<0.01)$, with the exception of voluntary activation $(P=0.1)$. No significant group $\times$ time interaction effects were demonstrated for any of the measures, although a strong trend was observed in the group $\times$ time interaction for knee pain $(P=0.053)$. The fact that only a trend emerged inhibited our ability to perform post hoc analysis to determine the source for the possible interaction effect. However, it seems this strong trend was primarily a result of the greater decrease in knee pain in the NMES group compared with in the exercise-only group, which occurred between the pre- and posttreatment assessments but leveled off by the follow-up assessment (Figure 2). Although ANOVA indicated no time, treatment, or interaction effects on voluntary activation, the mean improvement in the voluntary activation posttreatment in the NMES group was $22.2 \%$, whereas the mean improvement in the exercise group was only $9.6 \%$. Thus, although the improvement in the NMES group seemed to be more than double the improvement in the exercise-only group, these differences did not reach statistical significance $(P=0.1)$ (Figure 4).

The Tukey's post hoc analysis was employed to determine the source for the time effect for each outcome measure, with the exception of voluntary activation, where time effect did not reach significance (Table 4). The Tukey's post hoc analysis revealed significant improvements $(P<0.01)$ in all outcome measures between the pretest and posttest assessments. No significant differences between the posttest and the follow-up assessments were observed for pain, WOMAC, gait velocity, and stair negotiations. The significant differences for pain, WOMAC, and gait velocity between the pretest and follow-up assessment indicate these variables maintained improvements 12 weeks after completion of the program. In contrast, improvements were not maintained at the follow-up assessment for TUG, stair negotiation, and the MVIC normalized to the subject's body weight (W-MVIC). 


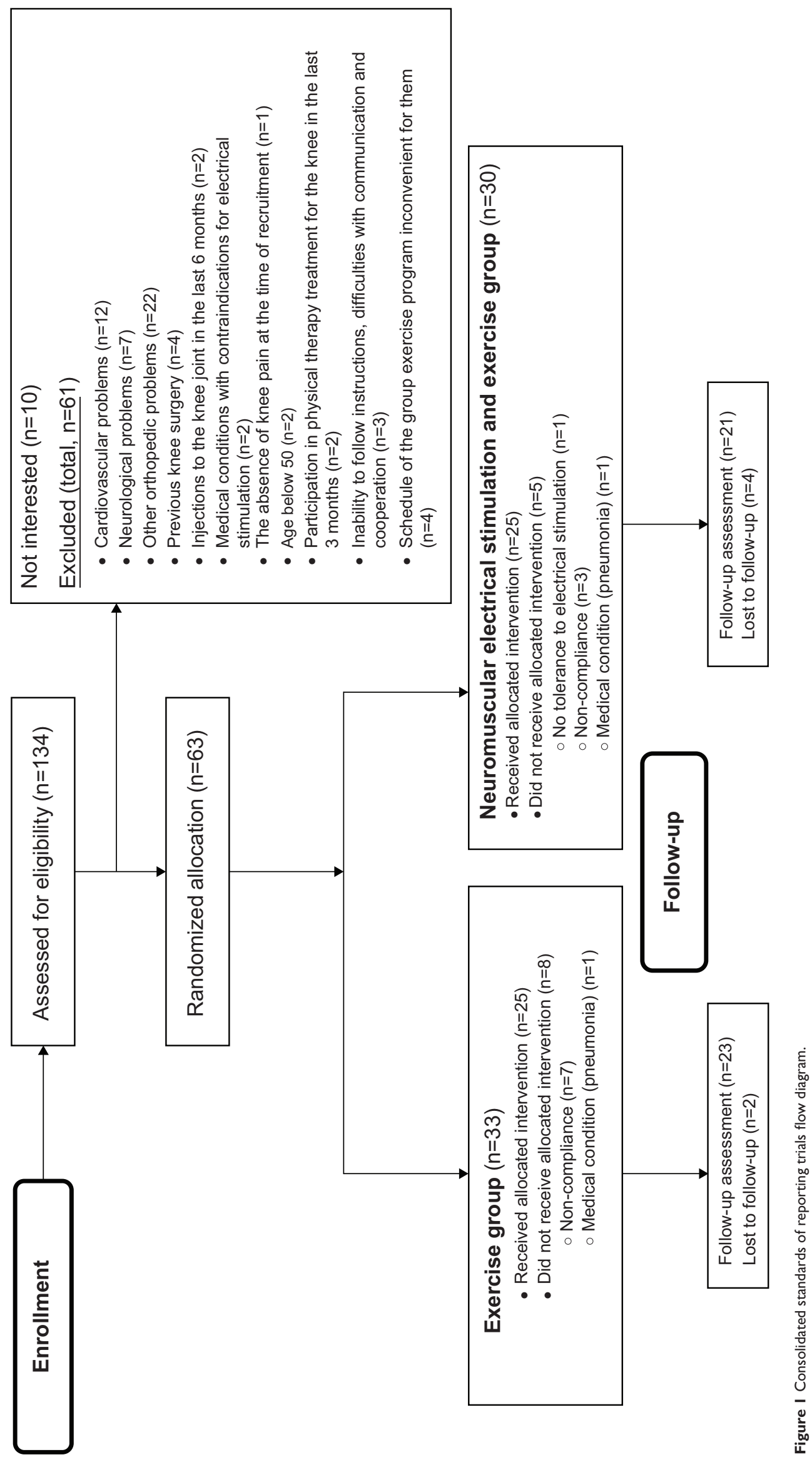


Table 2 Outcomes measures by group across assessments (mean [95\% confidence interval])

\begin{tabular}{|c|c|c|c|c|c|c|}
\hline \multirow[t]{2}{*}{ Variable } & \multicolumn{3}{|l|}{ Exercise group } & \multicolumn{3}{|c|}{ Exercise and NMES group } \\
\hline & Pretest $(n=25)$ & Posttest $(n=25)$ & Follow-up $(n=23)$ & Pretest $(n=25)$ & Posttest $(n=25)$ & Follow-up $(n=2 I)$ \\
\hline VAS, $0-10$ & $7.5(6.5-8.4)$ & $5(4.1-6)$ & $5.3(4.3-6.3)$ & $7.4(6.5-8.4)$ & $3.3(2.4-3.4)$ & $3.4(2.4-4.3)$ \\
\hline WOMAC, 0-240 & I08.I (88.2-128) & $75.6(55.7-95.5)$ & $80.2(59.1-101.3)$ & $99(79.1-118.9)$ & $52.4(32.5-54.4)$ & $65.5(45.2-85.9)$ \\
\hline $10 \mathrm{MW}$, seconds & $10.9(9.97-11.9)$ & $9.2(8.3-10.2)$ & $9.9(8.9-10.9)$ & $10.7(9.78-\mid 1.7)$ & $9.1(8.1-9.2)$ & $9.5(8.5-10.5)$ \\
\hline Stair, seconds & $19.6(16.4-22.8)$ & $16.6(\mid 3.4-19.9)$ & I8.2(|4.8-2|.6) & $20.1(16.9-23.3)$ & $16.4(13.1-16.7)$ & $17.9(|4.5-2| .3)$ \\
\hline TUG, seconds & $13(11.6-14.4)$ & $10.7(9.4-12.1)$ & $11.9(10.5-13.4)$ & $12.9(\mid 1.6-14.3)$ & $10.9(9.6-11.1)$ & $11.7(10.4-13.2)$ \\
\hline W-MVIC, kg & $0.18(0.15-0.20)$ & $0.2(0.17-0.23)$ & $0.17(0.14-0.19)$ & $0.18(0.15-0.21)$ & $0.2(0.18-0.21)$ & $0.18(0.15-0.21)$ \\
\hline VA, $\%$ & $64.4(52.4-76.3)$ & $69.12(55.6-82.7)$ & $63.1(49.6-76.6)$ & $69.7(60.8-78.7)$ & $85.1(75.5-86.1)$ & $70.3(60.4-80.2)$ \\
\hline
\end{tabular}

Abbreviations: NMES, neuromuscular electrical stimulation; VAS, visual analog scale; WOMAC, Western Ontario and McMaster Universities Osteoarthritis Index; I0 MW, $10 \mathrm{~m}$ walk test; TUG, timed up and go test; W-MVIC, maximal voluntary isometric contractions normalized to subject's weight; VA, voluntary activation.

Comparison between the pretest and follow-up assessments indicates that although performance in stair negotiation and W-MVIC regressed to the baseline values, TUG performance was still better at the follow-up assessment in comparison to the initial assessment.

\section{Discussion}

Consistent with previous studies demonstrating the positive effects of individualized exercise programs in subjects with knee $\mathrm{OA},{ }^{2,24}$ a comprehensive group exercise program had a significant positive effect on pain, functional ability, and QFM performance in individuals with knee OA. The results of the present study indicate that the addition of NMES to such a program enhanced the positive treatment effects primarily in terms of decreased knee pain. Although the reduction in knee pain observed in both treatment groups was maintained at the follow-up assessment, preference to the NMES treatment was not maintained at the follow-up assessment. Furthermore, the immediate positive effects on functional ability reported by the subjects (ie, WOMAC), as well as the gains in gait velocity, were maintained in both groups during a 12-week follow-up period, with the changes in WOMAC greater than the $20 \%$ change considered the minimum for clinically significant change. In contrast, the noted posttreatment improvements in TUG, stair negotiations, and muscle strength were not maintained at the follow-up assessments in either group.

Alleviating pain in subjects with knee OA is a critical issue, as it affects performance and quality of life and is often the primary reason for referral to physical therapy. ${ }^{25}$ Furthermore, knee pain often reduces subjects' adherence to prescribed exercise programs, resulting in a vicious cycle where pain results in increased muscle weakness and further increases in pain. ${ }^{12}$ Systematic reviews demonstrate inconsistent evidence regarding the effect of NMES on pain in subjects with knee OA. ${ }^{12,26}$ In contrast to the present study, Rosemffet et $\mathrm{al}^{27}$ report that a combination of NMES and exercise intervention is as effective as either

Pain intensity (VAS 0-10)

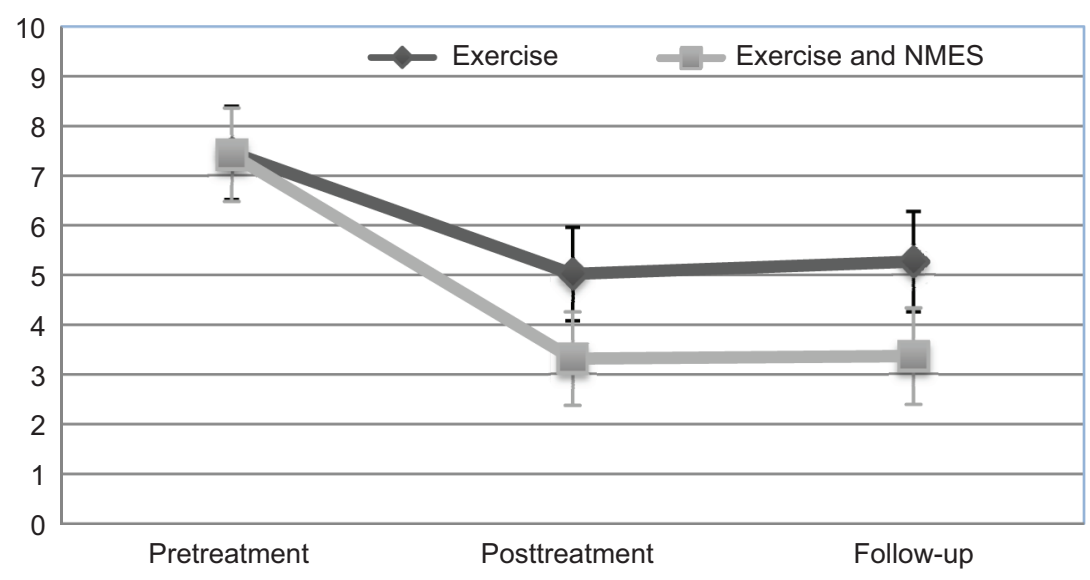

Figure 2 Knee pain intensity by group across assessments (mean and $95 \%$ confidence interval). Abbreviations: NMES, neuromuscular electrical stimulation; VAS, visual analog scale. 
WOMAC

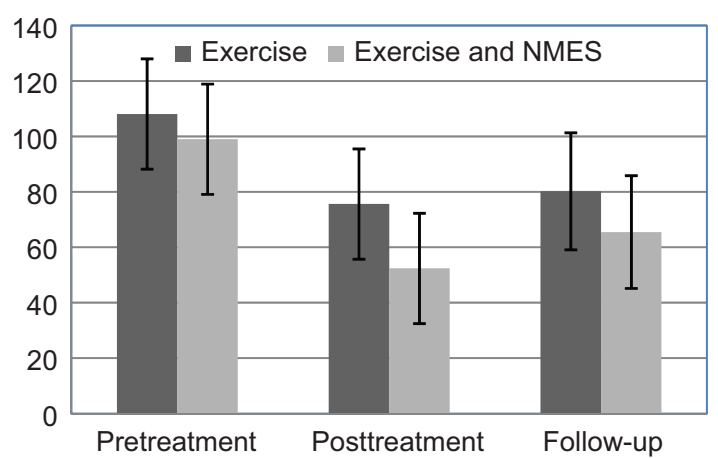

TUG (seconds)

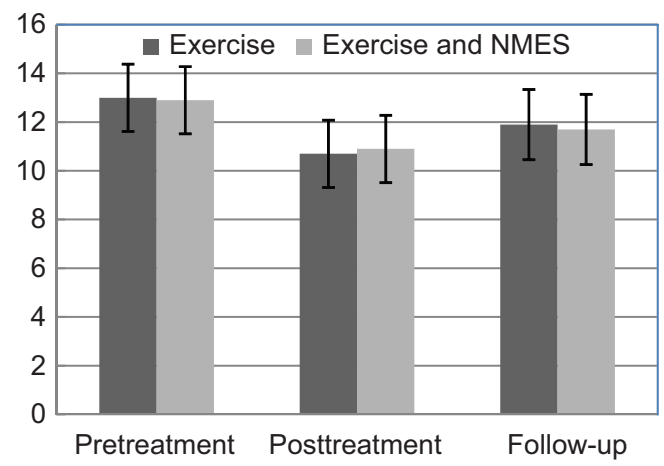

$10 \mathrm{MW}$ (seconds)

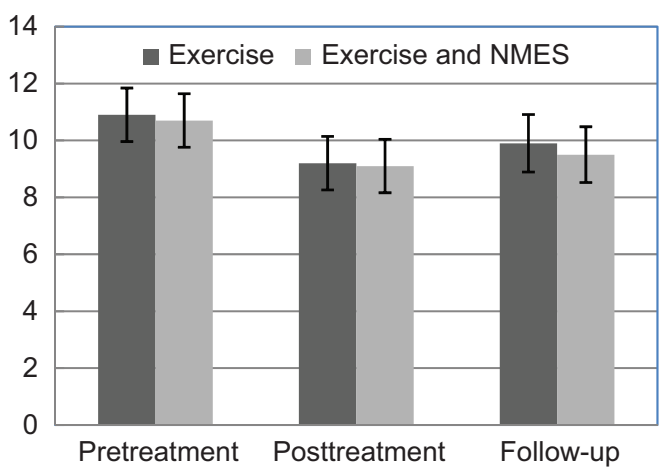

Stair negotiation (seconds)

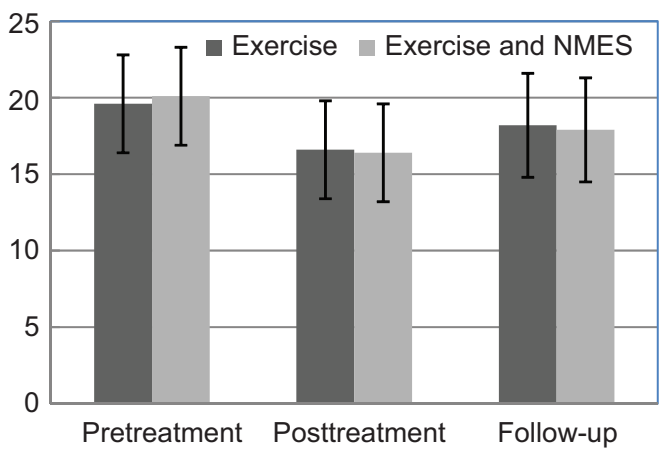

Figure 3 Physical ability measures by group across assessments (mean and $95 \%$ confidence interval).

Abbreviations: NMES, neuromuscular electrical stimulation; WOMAC, Western Ontario and McMaster Universities Osteoarthritis Index; I0 MW, I0m walk test; TUG, timed up and go test.

treatment alone in decreasing knee pain immediately posttreatment. Although no follow-up information was included in the study by Rosemffet et $\mathrm{al}^{27}$ both groups in the present study demonstrated a reduction in pain level that reached the minimal clinically significant difference of 2 points in the $\mathrm{VAS}^{28,29}$ and that was maintained 12 weeks after the intervention, although at this stage NMES had no additional advantage.

The precise mechanisms for the observed pain relief cannot be determined by this study. Although the electrical stimulation was set to induce muscle contractions, one cannot ignore the fact that such stimulation also provides intensive sensory input. Sensory stimulation has been shown to reduce peripheral and central sensitization and restore descending pain inhibition by the release of endogenous analgesics in subjects with chronic musculoskeletal pain. ${ }^{30}$ As exercise alone also stimulates the release of endogenous opioids, ${ }^{31,32}$ it is possible that the combination of NMES and exercise has a cumulative effect that explains the greater effect on pain observed in the NMES group immediately after treatment. The fact that the NMES effect on pain was lost in the follow-up is probably related to the temporary effects of nerve stimulation on pain alleviation.

Stair negotiation is a highly demanding functional task that is most frequently impaired in people with knee OA and persists even after total knee arthroplasty. ${ }^{10}$ Similar to TUG, it requires greater range of motion ${ }^{33,34}$ and knee muscle strength ${ }^{35}$ than level walking. The fact that muscle strength gains were not maintained over time may explain the regression in stair negotiation and TUG in the follow-up assessment.

The results of the current study failed to demonstrate that, as hypothesized, the addition of NMES to a group exercise program will further enhance the effect of exercise on QFM strength. Recent systematic reviews have demonstrated inconsistent results regarding the effect of NMES on QFM strength in patients with knee OA, whether applied as a single intervention or in combination with other therapeutic interventions. ${ }^{12,26}$ For example, NMES as a sole treatment had a significant positive effect in one study ${ }^{13}$ and demonstrated a trend toward such an effect in two additional studies that compared NMES with an exercise program, ${ }^{17,36}$ with only one 


\section{Voluntary activation (\%)}

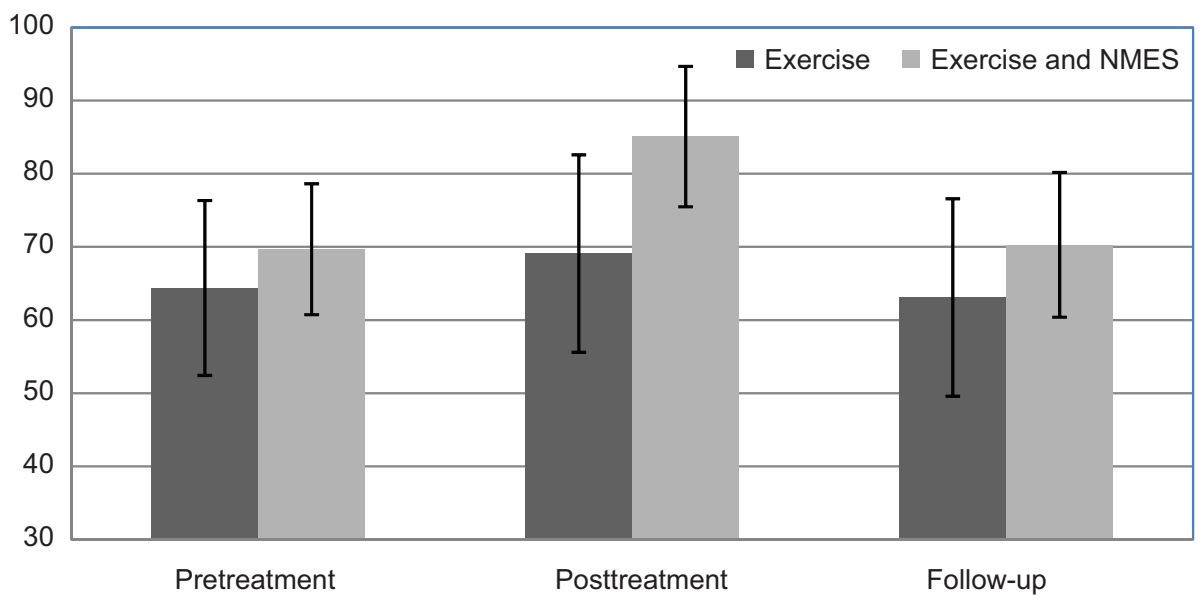

Figure 4 Voluntary activation of the quadriceps muscle by group across assessments (mean and $95 \%$ confidence interval). Abbreviation: NMES, neuromuscular electrical stimulation.

study demonstrating no change in force production. ${ }^{15}$ The only study that compared the effect of adding NMES to an exercise program supports our present findings by demonstrating muscle strength improvement in both groups, with no additive effect of NMES. ${ }^{27}$ The finding that gains in muscle strength were not maintained at the follow-up assessment in either treatment group is supported by a few previous studies that included follow-up assessments. ${ }^{16,36,37}$ They indicate that elderly individuals with chronic knee OA should probably adhere to an ongoing muscle strengthening routine that may help maintain gains in functional mobility.

The insignificant additive effect of NMES observed in this study might be related to two primary factors. It has been suggested that because the effect of NMES is dose-dependent, ${ }^{38,39}$ the lack of response may be a result of insufficient current intensity applied during stimulation or insufficient stimulation repetitions. In addition, the deficits in QFM resulting from OA are considerably less than those

Table 3 Results of analysis of variance comparing two interventions groups across time ( $P$-value)

\begin{tabular}{llll}
\hline Variable & Group effect & Time effect & Interaction \\
\hline VAS, 0-I0 & 0.02 & $0.000 I$ & 0.053 \\
WOMAC, 0-240 & 0.22 & 0.0001 & $0.5 \mathrm{I}$ \\
I0 MW, seconds & 0.67 & 0.0001 & 0.94 \\
Stair, seconds & 1.0 & $0.001 \mathrm{I}$ & 0.88 \\
TUG, seconds & 0.99 & $0.000 \mathrm{I}$ & 0.88 \\
W-MVIC, kg & 0.72 & 0.0001 & 0.71 \\
VA, \% & 0.1 & 0.1 & 0.56
\end{tabular}

Abbreviations: VAS, visual analog scale; WOMAC, Western Ontario and McMaster Universities Osteoarthritis Index; $10 \mathrm{MW}$, 10m walk test; TUG, timed up and go test; W-MVIC, maximal voluntary isometric contractions normalized to subject's weight; VA, voluntary activation. observed in other knee pathologies, such as anterior cruciate ligament reconstruction ${ }^{9,40}$ or total knee arthroplasty, ${ }^{10}$ which may have resulted in a ceiling effect provided by the exercise program. ${ }^{15,18}$

QFM weakness related to OA may stem from voluntary activation failure (ie, an inability to fully activate the QFM) and/or muscle atrophy. ${ }^{41}$ Accordingly, the gain in muscle strength that was observed in both groups may be attributed to improvement in either one of these factors. The current analyses, which included a follow-up assessment, did not identify a significant effect on voluntary activation of QFM in either group. In contrast, earlier analysis of the same data set, which included only the pre- and postassessment, ${ }^{18}$ demonstrated a significant change in neural recruitment $(P<0.05)$. Although no interaction effects between group and assessment time were observed in the present analysis, it should be noted that the immediate mean improvement in the voluntary activation in the NMES group was significantly greater than the immediate effect of an exercise-only treatment. The statistically insignificant results observed in the present analysis may be because the change in voluntary activation was not maintained in the follow-up assessment.

Improvements in voluntary activation of the QFM after an NMES training program have been demonstrated in young, healthy subjects ${ }^{42}$ and in individuals with other pathological knee conditions. ${ }^{10,40}$ To the best of our knowledge, only Palmieri-Smith et $\mathrm{al}^{37}$ assessed the immediate effect of NMES on voluntary activation of the QFM in subjects with knee OA and found no change in voluntary activation. The disparity between the current study and 
Table 4 Tukey's post hoc analysis comparing changes in the outcome measures across time (P-value)

\begin{tabular}{llll}
\hline Outcome measure & Pretest versus posttest & Posttest versus follow-up & Pretest versus follow-up \\
\hline VAS, 0-10 & 0.0001 & 0.93 & 0.0001 \\
WOMAC, 0-240 & 0.0001 & 0.36 & 0.0001 \\
IO MW, seconds & 0.0001 & 0.27 & 0.008 \\
Stair, seconds & 0.0007 & 0.23 & 0.14 \\
TUG, seconds & 0.0001 & 0.03 & 0.013 \\
W-MVIC, kg & 0.001 & 0.0001 & 0.66 \\
\hline
\end{tabular}

Abbreviations: VAS, visual analog scale; WOMAC, Western Ontario and McMaster Universities Osteoarthritis Index; 10 MW, I0m walk test; TUG, timed up and go test; W-MVIC, maximal voluntary isometric contractions normalized to subject's weight.

that of Palmieri-Smith et $\mathrm{a}^{37}$ may be because their subjects were basically asymptomatic in terms of pain, whereas the subjects in the current study all had a moderate to high degree of knee pain. Further studies are necessary to elucidate the relationships between treatment dose and changes in voluntary activation and strength recovery, as well as the relationship between initial deficits and effectiveness of interventions.

Several limitations to this study must be considered. Although the person conducting the exercise program was blinded to treatment allocation, blindness of the assessor was not maintained in the posttreatment and follow-up assessments. In addition, the electrically induced torques were not monitored throughout the treatment protocol. Although the patients were continuously encouraged to tolerate the highest possible current, it is possible that real-time information with visual feedback on the electrically induced force production would have encouraged and motivated the patients to tolerate higher current amplitude, thereby improving the effectiveness of the NMES treatment.

\section{Conclusion}

The addition of NMES to an exercise program has only a short time advantage in terms of knee pain modulation. Muscle strength and functional performance were equally improved posttreatment in patients receiving an exercise program alone or in combination with NMES treatment. The improvements in self-reported functional ability and on walk velocity were maintained at the follow-up assessment 12 weeks later. In contrast, the posttreatment improvements in the muscle strength, TUG, and stair negotiation tests were not maintained at the follow-up assessment. Further studies are needed to examine the effects of repeated bouts of exercise and NMES on the symptoms associated with knee OA.

\section{Disclosure}

The authors report no conflicts of interest in this work.

\section{References}

1. Michael JW, Schlüter-Brust KU, Eysel P. The epidemiology, etiology, diagnosis, and treatment of osteoarthritis of the knee. Dtsch Arztebl Int. 2010;107(9):152-162.

2. Bennell K, Hinman R. Exercise as a treatment for osteoarthritis. Curr Opin Rheumatol. 2005;17(5):634-640.

3. Hochberg MC, Altman RD, April KT, et al; American College of Rheumatology. American College of Rheumatology 2012 recommendations for the use of nonpharmacologic and pharmacologic therapies in osteoarthritis of the hand, hip, and knee. Arthritis Care Res (Hoboken). 2012;64(4):465-474.

4. O'Reilly SC, Jones A, Muir KR, Doherty M. Quadriceps weakness in knee osteoarthritis: the effect on pain and disability. Ann Rheum Dis. 1998;57(10):588-594.

5. Hurley MV. The role of muscle weakness in the pathogenesis of osteoarthritis. Rheum Dis Clin North Am. 1999;25(2):283-298, vi.

6. Slemenda C, Brandt KD, Heilman DK, et al. Quadriceps weakness and osteoarthritis of the knee. Ann Intern Med. 1997;127(2):97-104.

7. Pelland L, Brosseau L, Wells G, et al. Efficacy of strengthening exercises for osteoarthritis (Part I): A meta-analysis. Phys Ther Rev. 2004;9(2): 77-108.

8. Maffiuletti NA. Physiological and methodological considerations for the use of neuromuscular electrical stimulation. Eur J Appl Physiol. 2010;110(2):223-234.

9. Taradaj J, Halski T, Kucharzewski M, et al. The effect of neuromuscular electrical stimulation on quadriceps strength and knee function in professional soccer players: return to sport after ACL reconstruction. Biomed Res Int. 2013;2013:802534.

10. Monaghan B, Caulfield B, O’Mathúna DP. Surface neuromuscular electrical stimulation for quadriceps strengthening pre and post total knee replacement. Cochrane Database Syst Rev. 2010;(1):CD007177.

11. Fitzgerald GK, Piva SR, Irrgang JJ, Bouzubar F, Starz TW. Quadriceps activation failure as a moderator of the relationship between quadriceps strength and physical function in individuals with knee osteoarthritis. Arthritis Rheum. 2004;51(1):40-48.

12. Giggins O, Fullen B, Coughlan G. Neuromuscular electrical stimulation in the treatment of knee osteoarthritis: a systematic review and metaanalysis. Clin Rehabil. 2012;26(10):867-881.

13. Durmuş D, Alayli G, Cantürk F. Effects of quadriceps electrical stimulation program on clinical parameters in the patients with knee osteoarthritis. Clin Rheumatol. 2007;26(5):674-678.

14. Gaines JM, Metter EJ, Talbot LA. The effect of neuromuscular electrical stimulation on arthritis knee pain in older adults with osteoarthritis of the knee. Appl Nurs Res. 2004;17(3):201-206.

15. Palmieri-Smith RM, Thomas AC, Karvonen-Gutierrez C, Sowers M. A clinical trial of neuromuscular electrical stimulation in improving quadriceps muscle strength and activation among women with mild and moderate osteoarthritis. Phys Ther. 2010;90(10):1441-1452.

16. Talbot LA, Gaines JM, Ling SM, Metter EJ. A home-based protocol of electrical muscle stimulation for quadriceps muscle strength in older adults with osteoarthritis of the knee. $J$ Rheumatol. 2003;30(7): 1571-1578. 
17. Walls RJ, McHugh G, O’Gorman DJ, Moyna NM, O’Byrne JM. Effects of preoperative neuromuscular electrical stimulation on quadriceps strength and functional recovery in total knee arthroplasty. A pilot study. BMC Musculoskelet Disord. 2010;11(1):119.

18. Elboim-Gabyzon M, Rozen N, Laufer Y. Does neuromuscular electrical stimulation enhance the effectiveness of an exercise programme in subjects with knee osteoarthritis? A randomized controlled trial. Clin Rehabil. 2013;27(3):246-257.

19. Kellgren JH, Lawrence JS. Radiological assessment of osteo-arthrosis. Ann Rheum Dis. 1957;16(4):494-502.

20. Boonstra AM, Schiphorst Preuper HR, Reneman MF, Posthumus JB, Stewart RE. Reliability and validity of the visual analogue scale for disability in patients with chronic musculoskeletal pain. Int J Rehabil Res. 2008;31(2):165-169.

21. McConnell S, Kolopack P, Davis AM. The Western Ontario and McMaster Universities Osteoarthritis Index (WOMAC): a review of its utility and measurement properties. Arthritis Rheum. 2001;45(5): 453-461.

22. Laufer Y, Elboim M. Effect of burst frequency and duration of kilohertz-frequency alternating currents and of low-frequency pulsed currents on strength of contraction, muscle fatigue, and perceived discomfort. Phys Ther. 2008;88(10):1167-1176.

23. Shield A, Zhou S. Assessing voluntary muscle activation with the twitch interpolation technique. Sports Med. 2004;34(4):253-267.

24. Fransen M, McConnell S. Exercise for osteoarthritis of the knee. Cochrane Database Syst Rev. 2008;(4):CD004376.

25. Brakke R, Singh J, Sullivan W. Physical therapy in persons with osteoarthritis. PMR. 2012;4(5)(suppl):S53-S58.

26. de Oliveira Melo M, Aragão FA, Vaz MA. Neuromuscular electrical stimulation for muscle strengthening in elderly with knee osteoarthritis - a systematic review. Complement Ther Clin Pract. 2013;19(1):27-31.

27. Rosemffet MG, Schneeberger EE, Citera G, et al. Effects of functional electrostimulation on pain, muscular strength, and functional capacity in patients with osteoarthritis of the knee. J Clin Rheumatol. 2004;10(5): 246-249.

28. Pham T, van der Heijde D, Altman RD, et al. OMERACT-OARSI initiative: Osteoarthritis Research Society International set of responder criteria for osteoarthritis clinical trials revisited. Osteoarthritis Cartilage. 2004;12(5):389-399.

29. Tubach F, Ravaud P, Baron G, et al. Evaluation of clinically relevant changes in patient reported outcomes in knee and hip osteoarthritis: the minimal clinically important improvement. Ann Rheum Dis. 2005;64(1): 29-33.
30. Sluka KA, Walsh D. Transcutaneous electrical nerve stimulation: basic science mechanisms and clinical effectiveness. J Pain. 2003;4(3): $109-121$.

31. Sforzo GA. Opioids and exercise. An update. Sports Med. 1989; 7(2):109-124.

32. Wipfli BM, Rethorst CD, Landers DM. The anxiolytic effects of exercise: a meta-analysis of randomized trials and dose-response analysis. J Sport Exerc Psychol. 2008;30(4):392-410.

33. Riener R, Rabuffetti M, Frigo C. Stair ascent and descent at different inclinations. Gait Posture. 2002;15(1):32-44.

34. Rowe PJ, Myles CM, Walker C, Nutton R. Knee joint kinematics in gait and other functional activities measured using flexible electrogoniometry: how much knee motion is sufficient for normal daily life? Gait Posture. 2000;12(2):143-155.

35. Nadeau S, McFadyen BJ, Malouin F. Frontal and sagittal plane analyses of the stair climbing task in healthy adults aged over 40 years: what are the challenges compared to level walking? Clin Biomech (Bristol, Avon). 2003;18(10):950-959.

36. Bruce-Brand RA, Walls RJ, Ong JC, Emerson BS, O’Byrne JM, Moyna NM. Effects of home-based resistance training and neuromuscular electrical stimulation in knee osteoarthritis: a randomized controlled trial. BMC Musculoskelet Disord. 2012;13(1):118.

37. Palmieri-Smith RM, Thomas AC, Wojtys EM. Maximizing quadriceps strength after ACL reconstruction. Clin Sports Med. 2008;27(3) 405-424, vii-ix.

38. Marmon AR, Snyder-Mackler L. Quantifying Neuromuscular Electrical Stimulation Dosage after Knee Arthroplasty. J Life Sci (Libertyville). 2011;5(8):581-583

39. Snyder-Mackler L, Delitto A, Stralka SW, Bailey SL. Use of electrical stimulation to enhance recovery of quadriceps femoris muscle force production in patients following anterior cruciate ligament reconstruction. Phys Ther. 1994;74(10):901-907.

40. Fitzgerald GK, Piva SR, Irrgang JJ. A modified neuromuscular electrical stimulation protocol for quadriceps strength training following anterior cruciate ligament reconstruction. J Orthop Sports Phys Ther. 2003;33(9):492-501.

41. Stevens JE, Mizner RL, Snyder-Mackler L. Quadriceps strength and volitional activation before and after total knee arthroplasty for osteoarthritis. J Orthop Res. 2003;21(5):775-779.

42. Gondin J, Guette M, Ballay Y, Martin A. Electromyostimulation training effects on neural drive and muscle architecture. Med Sci Sports Exerc. 2005;37(8):1291-1299.
Clinical Interventions in Aging

\section{Publish your work in this journal}

Clinical Interventions in Aging is an international, peer-reviewed journal focusing on evidence-based reports on the value or lack thereof of treatments intended to prevent or delay the onset of maladaptive correlates of aging in human beings. This journal is indexed on PubMed Central, MedLine,

\section{Dovepress}

CAS, Scopus and the Elsevier Bibliographic databases. The manuscript management system is completely online and includes a very quick and fair peer-review system, which is all easy to use. Visit http://www.dovepress. com/testimonials.php to read real quotes from published authors. 